Original Article

\title{
PRESCRIPTION PATTERN AND USAGE OF ANTIMICROBIAL AGENTS FOR TREATING DIABETIC FOOT INFECTIONS AT TERTIARY CARE CENTRE
}

\author{
DASARAJU RAJESH ${ }^{1^{*}}$, M. V. ADVAITHA ${ }^{2}$, RAJENDRA HOLLA ${ }^{3}$ \\ ${ }^{1}$ Department of Pharmacology, GEMS and H, Srikakulam, Andhra Pradesh, India, ${ }^{2,3}$ Department of Pharmacology, KS Hegde Medical \\ Academy, Mangalore, Karnataka, India \\ Email: drdasaraju@gmail.com \\ Received: 08 May 2020, Revised and Accepted: 11 Jun 2020
}

\begin{abstract}
Objective: Due to the uncertainty about optimal antibiotic treatment, and probably substantial variation in practice, the present study was carried out to determine the bacterial profiles of infected diabetic foot ulcers (DFUs) and also to analyze the prescribing pattern of antibiotics used.

Methods: A prospective observational study was carried out in the department of General surgery at a tertiary care teaching hospital, Mangalore. Demographic details and treatment data of 78 patients were collected in a specially designed Proforma, and the data were analyzed using Microsoft Excel.

Results: According to Meggit-Wagner's classification, patients admitted with DFUs predominantly belonged to WAGNER 1 category (36\%), followed by WAGNER $4(26 \%)$ and WAGNER 2 (22\%) categories. Out of 66 culture-positive specimens, 21 (31.8\%) had monomicrobial flora, and 45 (68.2\%) had polymicrobial flora. A total of 148 organisms were obtained from the specimens. The most common isolates were Staphylococcus aureus $(22.3 \%)$ and Pseudomonas aeruginosa (17.5\%). Ceftriaxone was the most commonly prescribed empirical antibiotic (29\%), followed by linezolid (20\%), piperacillin-tazobactam (20\%), amoxicillin-clavulanic acid (13\%), cefoperazone-sulbactam (11\%). After the culture and sensitivity (C/S) results, antimicrobials were changed in $74.61 \%$ of patients in the preference of Linezolid (51\%), Amikacin (27\%), Levofloxacin (19\%), Ciprofloxacin (17\%), Piperacillin-tazobactam (13\%), Cefixime (15\%), Ceftriaxone $(11 \%)$ among others. Clindamycin and metronidazole were used to cover anaerobic microorganisms.
\end{abstract}

Conclusion: Most of the microorganisms isolated from DFUs were resistant to many types of antibiotics. Gram-positive organisms were largely sensitive to linezolid and vancomycin, while Gram-negative organisms to amikacin and imipenem. Local treatment of wounds is essential.

Keywords: Diabetic foot infections, Meggit-Wagner's classification, Bacterial profiles, Antibiotics

(C) 2020 The Authors. Published by Innovare Academic Sciences Pvt Ltd. This is an open access article under the CC BY license (http://creativecommons.org/licenses/by/4.0/) DOI: http://dx.doi.org/10.22159/ijpps.2020v12i8.38222. Journal homepage: https://innovareacademics.in/journals/index.php/ijpps.

\section{INTRODUCTION}

Diabetes mellitus (DM) is a serious chronic condition with devastating implications for affected patients across the globe. With little discrimination, it affects rich and poor, young and old, and industrialized or the economically less developed in equal measure. In 2011, the global prevalence of diabetes was 366 million (i.e.>8.3\% of the adult population across the globe). This fig. is predicted to reach 552 million by 2030 - a consequence of longer life expectancy, a sedentary lifestyle, and changing dietary patterns. The number of people with diabetes in India currently is 65.1 million, which is expected to rise to 142.7 million by 2035 unless urgent preventive steps are taken [1].

As the longevity of the diabetic population increases with the help of many safe and efficacious blood-sugar-lowering drugs, the incidence of diabetes-related complications, such as microvascular disease (nephropathy and retinopathy), neuropathy (peripheral and autonomic), peripheral arterial disease, and foot ulcer disease also rises. Among the serious complications of diabetes, disorders of the feet (ulceration, infection, gangrene, and amputation) are one of the most frequent causes of morbidity and are a leading cause of hospitalization of diabetic patients [2]. Approximately $25 \%$ of diabetic patients have a cumulative lifetime risk for foot ulcers, and these ulcers can get infected easily in $40-80 \%$ of the cases [3]. Only two-thirds of ulcers eventually heal; the remaining one-third is associated with progression and, if appropriate measures are not taken in time, could lead to systemic infection, septicemia, amputation (minor or major), or even death [4].

A wide range of bacteria (both aerobes and anaerobes) can cause infection in patients with chronic, long-standing DFUs. In recent years, the number of incidents and complications-related to DFIs has drastically increased due to the increased incidence of multidrug- resistant organisms (MDRO) [5]. Adequate management of these infections needs appropriate antibiotic selection on the basis of culture and susceptibility test reports [6]. Owing to severity and risk, it becomes imperative to initiate empirical antimicrobial treatment based on local epidemiological data of antimicrobial susceptibility without waiting for the results of culture and sensitivity. Each hospital should have antibiotic guidelines for the management of DFIs. The International Working Group on the Diabetic Foot (IWGDF) has published a comprehensive, evidencebased approach to the management of DFIs in an effort to standardize and improve care [7].

The well-established widely used Wagner-Meggitt wound classification system [8] and the new University of Texas (UT) diabetic wound classification system [9] both provide descriptions of ulcers to varying degrees. The Wagner system assesses ulcer depth and the presence of osteomyelitis or gangrene by using the following grades: grade 0 (intact Skin), grade 1 (superficial ulcer of the skin or subcutaneous tissue), grade 2 (ulcers extend into tendon, bone, or capsule), grade 3 (deep ulcer with osteomyelitis, or abscess), grade 4 (partial foot gangrene), and grade 5 (whole foot gangrene). The UT system assesses ulcer depth, the presence of wound infection, and the presence of clinical signs of lowerextremity ischemia by using the following grades: grade 0 (pre-or post-ulcerative site that has healed), grade 1 (superficial wound not involving tendon, capsule, or bone), grade 2 (wound penetrating to tendon or capsule), and grade 3 (wound penetrating bone or joint). Within each wound grade, there are four stages: clean wounds (stage A), nonischemic infected wounds (stage B), ischemic noninfected wounds (stage $C$ ), and ischemic infected wounds (stage D). Both wound classification systems are easy to use among health care providers, and both can provide a guide to planning treatment strategies. 
Although intensive efforts need to be made to avoid these foot complications, it is more easily said than done, especially in our country where walking barefoot is much more common than in the West [10]. Due to the high incidence of foot infections in diabetic patients and uncertainty about optimal antibiotic treatment, and probably substantial variation in practice, this study is taken up to help clinicians in prescribing antibiotics in light of recently published data.

\section{MATERIALS AND METHODS}

\section{Study design}

A prospective observational study was carried out on patients admitted with diabetic foot ulcers at Justice K S Hegde Charitable Hospital, Deralakatte, Mangalore, India. Seventy-eight patients were included in the study. The ethical clearance was obtained from the institutional ethical committee before the start of the study (INST. EC/E. C/004/2014-15). Informed consent was obtained from the patients in a prescribed form at the time of data collection.

\section{Data collection}

A specially designed proforma was developed to record the medical history, family history, examination details, antibiotic susceptibilities to isolated microorganisms, and antibiotic administration

\section{Inclusion criteria}

Patients with diabetes mellitus (previously diagnosed or newly diagnosed), presented with commonly and routinely noticed lower extremity infections.

\section{Exclusion criteria}

1. Diabetic foot ulcers with Wagner grade 0,1 , and patients on antibiotic therapy at the time of presentation.

2. Patients with foot infections due to any other causes such as nondiabetic-post-traumatic, arterial disorder alone, venous disorder alone, non-diabetic peripheral neuropathy, and secondary to implant infection.

3. Foot infections due to chronic diseases like tuberculosis, malignancy, and HIV.

4. Patients having an allergy to antimicrobial agents.

5. Diabetic foot infection in pregnancy.

\section{RESULTS}

A total of seventy-eight patients who were diagnosed to have diabetes mellitus with foot ulcers were selected for this study. Out of 78 patients, $65(83 \%)$ patients were male, and $13(17 \%)$ patients were female (fig. 1).

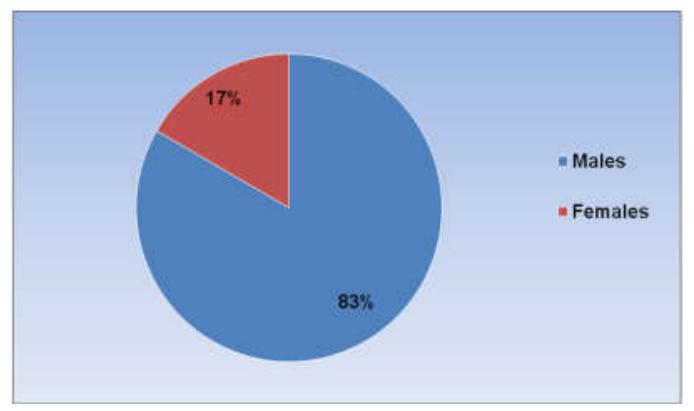

Fig. 1: Showing the genderwise distribution of patients

Most of the patients (65\%) belonged to the age group 51-70 y. More than half of the patients had diabetes mellitus for $>10 \mathrm{y}$. Diabetes was uncontrolled or poorly controlled $(\mathrm{HbA} 1 \mathrm{C}>8)$ in $67 \%$ of the cases. Essential hypertension was the most common associated comorbid condition seen in 32 patients $(68 \%)$. Most of the patients (n $=54,69 \%$ ) in the present study had foot ulcers for more than a onemonth duration. The majority of the diabetic foot ulcers belonged to WAGNER 1 category $(n=28,36 \%)$ followed by WAGNER $4(26 \%)$ and WAGNER $2(22 \%)$ categories (table 1$)$.
Out of 66 culture-positive specimens, $21 \quad(31.8 \%)$ had monomicrobial flora, and $45(68.2 \%)$ had polymicrobial flora. A total of 148 organisms were obtained from the specimens (table 2). There was no growth in specimens from 5 patients. The most common isolates in the present study were Staphylococcus aureus $(22.3 \%)$ and Pseudomonas aeruginosa (17.5\%). Gram-negative organisms dominated the gram-positive in case of the number of organisms isolated.

Table 1: Showing age, duration of diabetes, duration of foot ulcer, and ulcer severity in the patients $(n=78)$

\begin{tabular}{ll}
\hline Parameters & Number of patients (\%) \\
\hline Age duration (in years) & $1(1 \%)$ \\
$<40$ & $19(24 \%)$ \\
$41-50$ & $28(35 \%)$ \\
$51-60$ & $24(30 \%)$ \\
$61-70$ & $6(7 \%)$ \\
$>70$ & \\
Duration of diabetes mellitus (in years) & $26(33 \%)$ \\
$<10$ & $31(39 \%)$ \\
$11-20$ & $17(21 \%)$ \\
$>20$ & $4(5 \%)$ \\
Not known & \\
Duration of foot ulcer (in months) & $54(69 \%)$ \\
$>1$ mo & $24(31 \%)$ \\
Ulcer severity (as per wagner ulcer classification) & \\
1 & $28(36 \%)$ \\
2 & $17(22 \%)$ \\
4 & $9(11 \%)$ \\
5 & $20(26 \%)$ \\
\hline
\end{tabular}


Table 2: Profile of organisms isolated in patients with DFIs

\begin{tabular}{ll}
\hline Organisms & Number of organisms isolated (\%) \\
\hline Gram-positive organisms & $33(22.3 \%)$ \\
Staphylococcus aureus & $22(14.9 \%)$ \\
Enterococcus sp. & $2(1.3 \%)$ \\
Streptococcus sp. & $26(17.5 \%)$ \\
Gram-negative organisms & $23(15.5 \%)$ \\
Pseudomonas aeruginosa & $20(13.5 \%)$ \\
Escherichia coli & $11(7.4 \%)$ \\
Klebsiella sp. & $7(4.7 \%)$ \\
Acinetobacter & $1(0.6 \%)$ \\
Proteus sp. & $1(0.6 \%)$ \\
Enterobacter sp. & $1(0.6 \%)$ \\
Citrobacter sp. & $1(0.6 \%)$ \\
Providencia sp. & $148(100)$ \\
Morganella morganii & \\
Total
\end{tabular}

The sensitivity of the isolated aerobic bacteria to commonly used antibiotics is summarized in tables 3 and 4 , respectively. S. aureus showed maximum sensitivity to vancomycin and linezolid $(100 \%)$ followed by chloramphenicol (79\%), tetracycline (76\%), gentamicin $(76 \%)$, clindamycin $(73 \%)$, co-trimoxazole $(70 \%)$ respectively. Ten of the $33 \mathrm{~S}$. aureus isolates in this study were resistant to methicillin and were therefore considered as methicillin-resistant $S$. aureus
(MRSA). Enterococci were fully sensitive to ampicillin, vancomycin, and linezolid (100\%), followed by gentamicin and chloramphenicol (68\%). Gram-negative isolates were mostly sensitive to colistin and polymixin B $(100 \%)$ followed by imipenem, amikacin, gentamicin, piperacillin-tazobactam. $P$. aeruignosa has shown variable susceptibility towards commonly used antibiotics. Acinetobacter was totally resistant to most of the common antibiotics tested.

Table 3: In vitro activity of antimicrobial agents against Gram ${ }^{+v e}$ bacteria $(n=55)$

\begin{tabular}{lll}
\hline Antimicrobial agent & Staph aureus $(\boldsymbol{n}=\mathbf{3 3})$ & Enterococcus sp. (n=22) \\
\hline Ampicillin & & $22(100 \%)$ \\
Chloramphenicol & $26(79 \%)$ & $15(68 \%)$ \\
Clindamycin & $24(73 \%)$ & \\
Co-trimoxazole & $23(70 \%)$ & $11(50 \%)$ \\
Erythromycin & $18(55 \%)$ & $15(68 \%)$ \\
Gentamicin & $25(76 \%)$ & $22(100 \%)$ \\
Methicillin & $23(70 \%)$ & $9(41 \%)$ \\
Linezolid & $33(100 \%)$ & $22(100 \%)$ \\
Tetracycline & $25(76 \%)$ & \\
Vancomycin & $33(100 \%)$ & \\
\hline
\end{tabular}

Table 4: In vitro activity of antimicrobial agents against gram-ve bacteria $(n=87)$

\begin{tabular}{|c|c|c|c|c|c|}
\hline Antimicrobial agent & $\begin{array}{l}\text { Pseudomonas } \\
\text { aeruginosa }(n=26)\end{array}$ & $\begin{array}{l}\text { Escherichia coli } \\
(n=23)\end{array}$ & $\begin{array}{l}\text { Klebsiella sp. } \\
(n=20)\end{array}$ & Acinetobacter $(n=11)$ & $\begin{array}{l}\text { Proteus sp. } \\
(n=7)\end{array}$ \\
\hline \multicolumn{6}{|l|}{ Ampicillin } \\
\hline \multicolumn{6}{|l|}{ Amoxyclav } \\
\hline Cefuroxime & & $2(9 \%)$ & & & \\
\hline Cefotaxime & & $3(13 \%)$ & & & $7(100 \%)$ \\
\hline Ceftriaxone & & $5(22 \%)$ & & & $7(100 \%)$ \\
\hline Ceftazidime & $9(35 \%)$ & $5(22 \%)$ & & & $7(100 \%)$ \\
\hline Cefepime & $9(35 \%)$ & $6(26 \%)$ & $5(25 \%)$ & & $7(100 \%)$ \\
\hline Chloramphenicol & & $19(83 \%)$ & $9(45 \%)$ & & $5(71 \%)$ \\
\hline Gentamicin & $17(65 \%)$ & $13(57 \%)$ & $13(65 \%)$ & $1(9 \%)$ & \\
\hline Amikacin & $20(77 \%)$ & $18(78 \%)$ & $15(75 \%)$ & & $7(100 \%)$ \\
\hline Ciprofloxacin & $7(27 \%)$ & $6(26 \%)$ & $8(40 \%)$ & & $6(86 \%)$ \\
\hline Levofloxacin & $12(46 \%)$ & $6(26 \%)$ & $6(30 \%)$ & $3(27 \%)$ & $6(86 \%)$ \\
\hline Co-trimoxazole & & $11(48 \%)$ & $10(50 \%)$ & & $4(57 \%)$ \\
\hline PipTaz & $10(38 \%)$ & $12(52 \%)$ & $9(45 \%)$ & $9(82 \%)$ & $7(100 \%)$ \\
\hline Cef-S & & $9(39 \%)$ & & & $7(100 \%)$ \\
\hline Imipenem & $19(73 \%)$ & $17(74 \%)$ & $18(90 \%)$ & $4(36 \%)$ & $7(100 \%)$ \\
\hline Tobramycin & $14(54 \%)$ & & & & \\
\hline Colistin & $26(100 \%)$ & $23(100 \%)$ & $20(100 \%)$ & $11(100 \%)$ & \\
\hline Polymixin B & $26(100 \%)$ & $23(100 \%)$ & $20(100 \%)$ & $11(100 \%)$ & \\
\hline
\end{tabular}

Amoxyclav-Amoxycillin+Clavulanic acid; PipTaz-Piperacillin+Tazobactam; Cef-S-Cefoperazone+Sulbactam

Conservative treatment was given in $30 \%$ of patients, whereas $70 \%$ of patients underwent surgical intervention (fig. 2). All 78 patients received empirical antimicrobials (table 5). Ceftriaxone was the most commonly prescribed antibiotic $(29 \%)$, followed by linezolid 
(20\%), piperacillin-tazobactam (20\%), amoxicillin-clavulanic acid (13\%), cefoperazone-sulbactam (11\%). After the C/S results, antimicrobials were changed in $74.61 \%$ of patients in the preference of linezolid (51\%), amikacin (27\%), levofloxacin (19\%), ciprofloxacin (17\%), piperacillin-tazobactam (13\%), cefixime (15\%), ceftriaxone $(11 \%)$ among others. Clindamycin and metronidazole were used to cover anaerobic microorganisms when infection was of a severe nature like septicemia and gangrene.

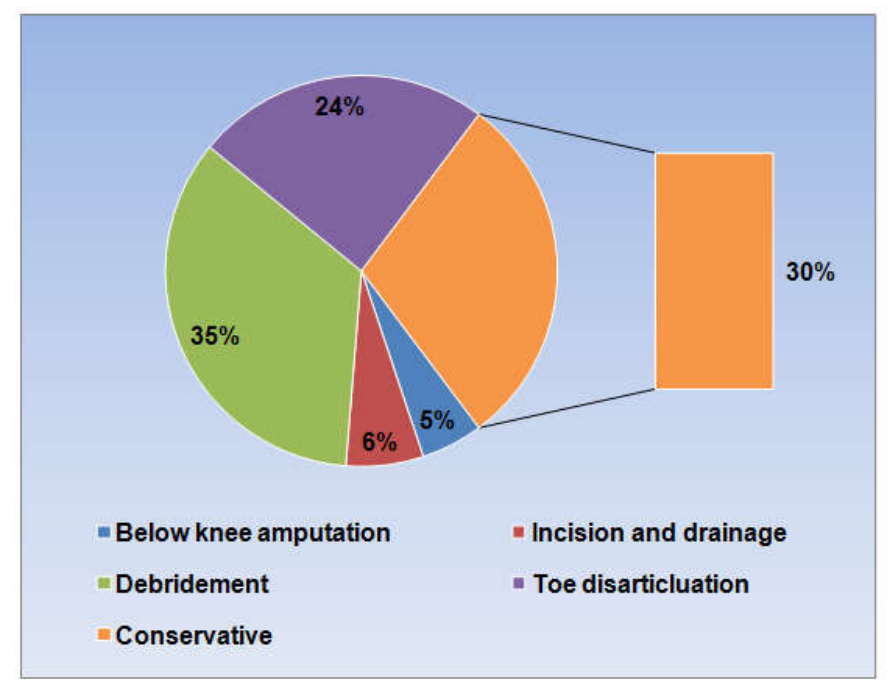

Fig. 2: Showing management choice of DFIs

Table 5: Spectrum of empirical antimicrobials employed

\begin{tabular}{ll}
\hline Antimicrobials & Number of patients (\%) \\
\hline Ceftriaxone & $23(29 \%)$ \\
Cefoperazone-sulbactam & $9(11 \%)$ \\
Cefepime & $4(5 \%)$ \\
Cefixime & $4(5 \%)$ \\
Cefuroxime & $1(1 \%)$ \\
Clindamycin & $1(1 \%)$ \\
Amoxyclav & $10(13 \%)$ \\
Linezolid & $16(20 \%)$ \\
Metronidazole & $11(14 \%)$ \\
Ornidazole & $10(13 \%)$ \\
Piperacillin-tazobactam & $16(20 \%)$ \\
\hline
\end{tabular}

\section{DISCUSSION}

A diabetic foot is one of the most feared complications of diabetes, and it is the leading cause of hospitalization among diabetic patients Baseline characteristics of the 78 diabetic foot infection patients taken for the study showed $83 \%(65 / 78)$ were males, and $17 \%(13 / 78)$ were females. Increased male prevalence has been reported in other studies [11]. This may be due to higher levels of outdoor activity among males than females. Most of the patients belong to the age group 51-60 y, which is similar to the study conducted by Gregg EW, et al. [12]. Duration of diabetes in most of the patients was $>10 \mathrm{y}$, which is in correlation with the study conducted by Gadepally, et al. [11].

Blood glucose was poorly controlled in 52 (67\%) patients. Essential hypertension was the most common associated co-morbid condition seen in 32 patients. A similar situation has been found in a study done by Jyothylekshmy, et al., where $76.89 \%$ of study patients admitted with recent and recurrent foot complications had systemic hypertension [13]. Nine out of 78 patients gave a history of alcohol intake, and 11 patients were cigarette smokers.

Most of the patients in the present study had foot ulcers for $>1$ month duration, which is in correlation with the study conducted by Sharma, et al. [14]. Forty-two patients had right foot ulcers (54\%) $30(38 \%)$ had ulcers on left foot, and the remaining $6(8 \%)$ patients had ulcers on the bilateral foot. The majority of the diabetic foot ulcers belonged to WAGNER 1 category $(n=28,36 \%)$ followed by WAGNER $4(26 \%)$ and WAGNER $2(22 \%)$ categories. Ten patients $(13 \%)$ had a previous history of minor amputation. Five patients (6\%) underwent split skin grafting before the time of admission.

In the present study, microbiological evaluation of diabetic foot ulcer infections showed polymicrobial growth in 63\% (44/70), monomicrobial growth in $30 \%(21 / 70)$, and no growth in $7 \%$ $(5 / 70)$. A similar pattern observed in several studies conducted both in India and abroad [15]. In contrast to the previous studies conducted in patients with DFI, which showed a predominance of gram-positive aerobes [16], recent studies conducted in India and elsewhere have shown a preponderance of gram-negative aerobes [17]. The present study also shows the changing trend in the organisms causing diabetic foot infections, with gram-negative bacteria replacing gram-positive bacteria as commonest agents $(91$ vs. 57). Similar to a study done by Peter, et al.[18], Staphylococcus aureus (33/148) was the commonest organism isolated on pus culture in the present study. In contrast, Pseudomonas and E. coli were the most common bacterial isolates in the studies conducted by Bansal, et al., and Tiwari, et al., respectively $[15,19]$. This kind of discrepancy could be because of geographical variations or the types and severity of infection included in the studies. 
Staphylococcus aureus showed maximum sensitivity to vancomycin and linezolid (100\%) followed by chloramphenicol (79\%), tetracycline $(76 \%)$, gentamicin $(76 \%)$, clindamycin $(73 \%)$, cotrimoxazole $(70 \%)$ respectively. Ten $(30 \%)$ of the 33 S. aureus isolates in this study were resistant to methicillin and were therefore considered as MRSA. The prevalence of MRSA in this study was found to be much lower than the study conducted by Tentolouris, et al. (50\% of $S$. aureus isolates were MRSA) [20]. MRSA isolates were mostly susceptible to linezolid and tetracycline and totally resistant to ciprofloxacin. However, some previous studies reported sensitivity to ciprofloxacin [15]. Enterococci were fully sensitive to ampicillin, vancomycin, and linezolid (100\%), followed by gentamicin and chloramphenicol $(68 \%)$.

Gram-negative isolates were mostly sensitive to colistin and polymixin B (100\%) followed by imipenem, amikacin, gentamicin, piperacillin-tazobactam. The majority of isolates of $E$. coli and $K$. pneumoniae were susceptible to amikacin and imipenem but were showing variable susceptibility to co-trimoxazole, gentamicin, and piperacillin-tazobactam. Six out of 23 samples containing E. coli showed resistance to imipenem. This is in contrast to a study done by Akhi, et al. [21] where all E. coli strains isolated were imipenem sensitive. Most studies have reported varying resistance patterns of $P$. aeruginosa toward commonly used antibiotics [15]. In the present study majority of isolates of $P$. aeruignosa were showing variable susceptibility to amikacin, ceftazidime, cefepime, gentamicin imipenem, levofloxacin, piperacillin-tazobactam, and tobramycin Acinetobacter $s p$. were totally resistant to most of the common antibiotics tested. This was in accordance with multidrug-resistant Acinetobacter isolates from Bansal, et al. study [15]. It was sensitive to imipenem, piperacillin-tazobactam, colistin, and polymixin B. Most of the isolated gram-negative bacteria (except for acinetobacter) are sensitive to amikacin, which is in accordance with a study done by Raja NS [22]. This may be due to the decrease in the use of amikacin in diabetic patients because of its nephrotoxic effect.

Conservative treatment was given in $30 \%$ of patients, whereas $70 \%$ of patients underwent surgical intervention. All 78 patients received empirical antimicrobials. In patients with DFUs having no and/or mild clinical signs of infection, oral antibiotics were the mainstay in the management of diabetic foot ulcers. Among oral antibiotics, amoxycillin/clavulanic acid was most commonly prescribed. After culture and sensitivity, linezolid was the most commonly prescribed antibacterial agent, since most common isolated pathogen in DFUs with mild clinical signs of infection include $S$. aureus. Although current IDSA guidelines recommend antibiotic treatment be initiated when obvious clinical signs of infection develop, these signs may not appear until the destruction of underlying tissue and bone triggers a systemic inflammatory response. Patients with diabetes, however, may not express clinical signs of infection, despite high levels of bacteria in local DFU tissue, because peripheral vascular disease, poor metabolic control, and neuropathy dampen first-line inflammatory responses. Therefore, in DFUs that show no clinica signs of infection, the judicious and timelier use of antimicrobial treatment might be useful. But for patients with uninfected DFUs, indiscriminate use of antibiotics likely contributes to the growing problem of antibiotic resistance.

In patients with DFUs having moderate to severe clinical signs of infection, parenteral antibiotics were administered. Ceftriaxone and piperacillin-tazobactam were the most commonly prescribed empirical antibacterial agents in the present study because of the predominance of gram-negative organisms isolated. Where infected DFUs were foul-smelling, the above agents are often combined with anti-anaerobic agents such as metronidazole or ornidazole and clindamycin. Linezolid was also prescribed along with anti-anaerobic agents in some patients where the clinical signs of infection appeared to be of moderate severity, in which case the suspected organism was MRSA. One patient with severe diabetic foot infection due to MDR $P$. aeruginosa post antimicrobial susceptibility test received colistin after other ineffective antimicrobial treatment

Surgery is the cornerstone of treating many deep soft tissue infections, and early intervention may be associated with better outcomes. Bone resection and amputation are often necessary when there is extensive soft tissue necrosis or to provide a more functional foot. In the present study, 26 (35\%) patients underwent debridement, and $20(24 \%)$ patients underwent minor amputation. Below knee, amputation was done in $4(5 \%)$ patients.

Using topical antibiotic therapy for a foot wound is appealing, as it allows high concentrations at the site of infection without potentially toxic systemic levels. It would also allow treatment with agents not available for systemic therapy. A limited number of marketed topical antimicrobial agents, as well as antimicrobial impregnated wound dressings [e. g., those containing various forms of silver and iodine]) might be useful for preventing or possibly even treating mild infections [23]. But the silver resistance is a documented problem with MRSA [24]. For deep surgical wounds, antibiotic-impregnated beads, cement, or biodegradable bovine collagen sponges can supply high local antibiotic concentrations (for a few days), and in some instances, fill dead space [25]. In the present study, topical agents were prescribed to 26 patients. Most commonly prescribed topical preparation include Amorphous Hydrogel Wound Dressing with Colloidal Silver Ointment (13 patients), Mupirocin Ointment (6 patients), Chlorhexidine acetate Cream (3 patients) and remaining 4 patients were prescribed Clindamycin phosphate Gel, Recombinant human platelet-derived growth factor Gel, Sodium pyrrolidone carboxylic acid+Sodium lactate+Olive oil Lotion, and Bacitracin zinc+Neomycin sulfate+Polymyxin B Ointment respectively.

\section{LIMITATIONS}

Diabetic foot infections are a heterogeneous group of infections in a diverse patient population. Therefore it is difficult to determine common optimal management and common optimal duration of antibiotic administration for the entire population.

\section{CONCLUSION}

Both Gram-positive cocci and Gram-negative bacilli caused diabetic foot infections, and this study showed a preponderance of Gramnegative bacilli. However, Staphylococcus aureus was the most predominant organism isolated from the lesions. The difference in the microbial pattern of diabetic foot infection in various studies shows that the empirical therapy in each case should be selected considering the most common specific pathogen of the region and its antimicrobial susceptibility. Because specimens from many patients with diabetic foot infections have polymicrobial cultures, empirical therapy should be relatively broad spectrum, especially for patients with severe infections and those who are immunocompromised. Despite it being the most feared complication, ignorance about diabetic foot continues to dominate the reasons for many of the avoidable limb amputations. Patient education, proper care of feet, and appropriate footwear can prevent the occurrence of many of the foot ulcerations [26]. Good glycaemic control is indeed very much essential.

\section{ACKNOWLEDGMENT}

I would like to thank the Head of Department, Dr. Rajendra Holla, for his core guidance and Dr. MV Advaitha for his constant support throughout the conduct of the study. I also extend my gratitude to the Head of Department General surgery for providing access to the patient data for the study.

\section{FUNDING}

Nil

\section{AUTHORS CONTRIBUTIONS}

The corresponding author designed the study, collected date from the medical records, and wrote the manuscript. Author MV Advaitha assisted in data collection from the medical records. Author Rajendra Holla guided in designing the study protocol.

\section{CONFLICT OF INTERESTS}

Declared none 


\section{REFERENCES}

1. Whiting DR, Guariguata L, Weil C, Shaw J. IDF diabetes atlas: global estimates of the prevalence of diabetes for 2011 and 2030. Diabetes Res Clin Pract 2013;94:311-21.

2. Ramsey SD, Newton K, Blough D, McCulloch DK, Sandhu N, Reiber GE, et al. Incidence, outcomes, and cost of foot ulcers in patients with diabetes. Diabetes Care 1999;22:382-7.

3. Singh N, Armstrong DG, Lipsky BA. Preventing foot ulcers in patients with diabetes. JAMA 2005;293:217-28.

4. Ang C, Lim Y. Recurrent admissions for diabetic foot complications. Malays Orthop J 2013;7:21-6.

5. Khoharo HK, Ansari S, Qureshi F. Diabetic foot ulcers: common isolated pathogens and in vitro antimicrobial activity. Prof Med J 2009;16:53-60.

6. Mansilha A, Brandão D. Guidelines for treatment of patients with diabetes and infected ulcers. J Cardiovasc Surg (Torino) 2013;54:193-200

7. Lipsky BA, Aragon Sanchez J, Diggle M. IWGDF guidance on the diagnosis and management of foot infections in persons with diabetes. Diabetes Metab Res Rev 2016;32 Suppl 1:45-74.

8. Wagner FW Jr. The dysvascular foot: a system for diagnosis and treatment. Foot Ankle 1981;2:62-122.

9. Armstrong DG, Lavery LA, Harkless LB. Validation of a diabetic wound classification system. The contribution of depth, infection, and ischemia to risk of amputation. Diabetes Care 1998;21:855-9.

10. Jayasinghe SA, Atukorala I, Gunethilleke B, Siriwardena V, Herath SC, De Abrew K. Is walking barefoot a risk factor for diabetic foot disease in developing countries? Rural Remote Health 2007;7:692.

11. Gadepalli R, Dhawan B, Sreenivas V, Kapil A, Ammini AC, Chaudhry R. A clinico-microbiological study of diabetic foot ulcers in an Indian tertiary care hospital. Diabetes Care 2006;29:1727-32.

12. Gregg EW, Sorlie P, Paulose Ram R, Gu Q, Eberhardt MS, Wolz $\mathrm{M}$, et al. prevalence of the lower-extremity disease in the US adult population $>=40 \mathrm{y}$ of age with and without diabetes: 1999-2000 national health and nutrition examination survey. Diabetes Care 2004;27:1591-7.

13. Jyothylekshmy V, Menon AS, Abraham S. Epidemiology of diabetic foot complications in a podiatry clinic of a tertiary hospital in South India. Indian J Health Sci 2015;8:48-51.

14. Sharma VK, Khadka PB, Joshi A, Sharma R. Common pathogens isolated in diabetic foot infection in Bir hospital. Kathmandu University Med J 2006;4:295-301.

15. Bansal E, Garg A, Bhatia S, Attri AK, Chander J. Spectrum of microbial flora in diabetic foot ulcers. Indian J Pathol Microbiol 2008;51:204-8.
16. Citron DM, Goldstein EJ, Merriam CV, Lipsky BA, Abramson MA. Bacteriology of moderate to severe diabetic foot infections and in vitro activity of antimicrobial agents. J Clin Microbiol 2007;45:2819-28.

17. Shankar EM, Mohan V, Premlatha G, Srinivasan RS, Usha AR. Bacterial etiology of diabetic foot infections in South India. Eur J Intern Med 2005;16:567-70

18. Peter N, Cherian N, Thomas S, George S, Sundresh JN. Study of prescribing pattern and use of antibiotics in the management of wound infection. Asian J Pharm Clin Res 2017;10:210-3.

19. Tiwari S, Pratyush DD, Dwivedi A, Gupta SK, Rai M, Singh SK. Microbiological and clinical characteristics of diabetic foot infection in northern India. J Infect Dev Ctries 2012;6:329-32.

20. Tentolouris N, Petrikkos G, Vallianou N, Zachos C, Daikos GL, Tsapogas $\mathrm{P}$, et al. Prevalence of methicillin-resistant staphylococcus aureus in infected and uninfected diabetic foot ulcers. Clin Microbiol Infect 2006;12:186-9.

21. Akhi MT, Ghotaslou R, Asgharzadeh M, Varshochi M, Pirzadeh $\mathrm{T}$, Memar MY, et al. Bacterial etiology and antibiotic susceptibility pattern of diabetic foot infections in tabriz, Iran. GMS Hyg Infect Control 2015;10:Doc02. DOI:10.3205/ dgkh000245

22. Raja NS. Microbiology of diabetic foot infections in a teaching hospital in Malaysia: a retrospective study of 194 cases. J Microbiol Immunol Infect 2007;40:39-44.

23. Lipsky BA, Hoey C. Topical antimicrobial therapy for treating chronic wounds. Clin Infect Dis 2009;49:1541-9.

24. Chopra I. The increasing use of silver-based products as antimicrobial agents: a useful development or a cause for concern? J Antimicrob Chemother 2007;59:587-90.

25. Roeder B, Van Gils CC, Maling S. Antibiotic beads in the treatment of diabetic pedal osteomyelitis. J Foot Ankle Surg 2000;39:124-30.

26. BB D, Unnikrishnan B, Kulkarni V, Thapar R, Mithra P, Kumar N, et al. Knowledge and practice regarding foot care among type 2 diabetes mellitus patients at a tertiary care hospital in coastal South India. Asian J Pharm Clin Res 2015;8:175-8. 